\title{
Research Article \\ Group Classification and Conservation Laws of a Class of Hyperbolic Equations
}

\author{
J. C. Ndogmo \\ Department of Mathematics and Applied Mathematics, University of Venda, P/B X5050, Thohoyandou 0950, South Africa \\ Correspondence should be addressed to J. C. Ndogmo; ndogmoj@yahoo.com
}

Received 1 May 2021; Revised 9 June 2021; Accepted 14 July 2021; Published 6 August 2021

Academic Editor: Victor Kovtunenko

Copyright @ 2021 J. C. Ndogmo. This is an open access article distributed under the Creative Commons Attribution License, which permits unrestricted use, distribution, and reproduction in any medium, provided the original work is properly cited.

\begin{abstract}
A method for the group classification of differential equations is proposed. It is based on the determination of all possible cases of linear dependence of certain indeterminates appearing in the determining equations of symmetries of the equation. The method is simple and systematic and applied to a family of hyperbolic equations. Moreover, as the given family contains several known equations with important physical applications, low-order conservation laws of some relevant equations from the family are computed, and the results obtained are discussed with regard to the symmetry integrability of a particular class from the underlying family of hyperbolic equations.
\end{abstract}

\section{Introduction}

The group classification of differential equations, which consists in determining all symmetry classes admitted by an equation according to the values of the parameters or arbitrary functions labelling the given family of differential equations, has been carried out in the literature mostly in a more or less ad hoc manner [1-7]. This has resulted, as pointed out in [7], in a number of revised classification results published in the literature being wrong. Attempts to find a somewhat systematic method for this classification problem has, however, been made. This includes the well-known algebraic method which can be traced back to Lie's work on symmetry algebras of ordinary differential equations (ODEs), and which has been upgraded or applied in several papers $[2,4$, $8-11]$. Another classification method that has emerged in recent years, often called furcate splitting, originated in [12], and its refinements and generalizations have been applied in other papers (see [13-15] and the references therein). Other methods suggested in the literature tend to be variants of the direct method, or adapted to a specific type of equations such as the one proposed for linear pdes in [6].

Some of the suggested methods still require, in general, a considerable amount of computations and analysis even for simpler cases of equations, or they are limited by the reduced scope of their applicability. For instance, the algebraic method can only be carried out when any symmetry algebra of the given family of nonlinear equations falls, in general, within the existing classification of the low-dimensional Lie algebras, in addition to other conditions. It should also be noted that the algebraic method can be complemented with other methods as done in $[14,16,17]$.

In this paper, we propose a new and systematic method for the group classification of differential equations. It is based on the determination of all possible cases of linear dependence of certain indeterminates appearing in the determining equations for the Lie point symmetry algebra of the given family of equations. The method is presented through its application to the family of scalar hyperbolic equations

$$
u_{x y}=F\left(\pi_{1} u+\pi_{2} u_{x}\right),
$$

labelled by the arbitrary function $F=F\left(\pi_{1} u+\pi_{2} u_{x}\right)$, where $u=u(x, y)$ and $\pi_{1}, \pi_{2}$ are scalars. Moreover, a variable in a subscript denotes a partial derivative with respect to the variable, so that $u_{x}=\partial u / \partial x, u_{y}=\partial u / \partial y, u_{x y}=\partial^{2} u /(\partial x \partial y)$, 
and so on. This systematic method turns out to be simple, algorithmic, and yields a relatively fast classification of the family (1) of equations.

Given that the family (1) contains several well-known equations with important physical applications, including amongst others Liouville's equation [18-20] and the sineGordon equation, low-order conservation laws of a number of relevant equations from this family are computed using the direct method [21-24] or some other methods [25]. Indeed, the sine-Gordon equation occurs for instance in the study of surfaces of constant negative curvature as well as in the study of crystal dislocations, and its solutions possess some soliton properties $[26,27]$. Liouville's equation on the other hand appears in the study of isothermal coordinates in classical mechanics and statistical physics [28]. More generally, as pointed out in [29], hyperbolic equations describe a large and important collection of phenomena. This includes aerodynamic, fluid, and atmospheric flows, amongst others. Moreover, their solutions tend to be more complex and more interesting than those of their parabolic or hyperbolic counterparts [29].

Some of our computations of conservation laws correspond to the case of arbitrary labelling functions of subclasses of (1). Although some of the conservation laws found are finite in number, they are infinite for other equations, and the connection between such equations and their symmetry integrability is discussed. It will naturally be assumed that $F$ is a nonconstant function.

\section{Equivalence Group}

In order to ease the classification problem of (1), as usual, we shall make use of its group of equivalence transformations. The complete determination of this group is summarized in the following result.

Theorem 1. The group of equivalence transformations of (1) is given as follows, where $w=w(t, z)$ is the new dependent variable.

Case $1 . \pi_{2}=0$. The equation reduces in this case to

$$
u_{x y}=F(u)
$$

and the invertible point transformations are given by

$$
\begin{aligned}
& x=a t+b, \\
& y=c z+d, \\
& u=r w+s,
\end{aligned}
$$

or

$$
\begin{aligned}
& x=a z+b, \\
& y=c t+d, \\
& u=r w+s,
\end{aligned}
$$

for some arbitrary constants $a, b, c, d, r, s$, with acr $\neq 0$. The corresponding transformed equation has the following expression:

$$
w_{t z}=H(w)
$$

where $H=(a c / r) F(r w+s)$.

Case 2. $\pi_{1}=0$. The equation reduces in this case to

$$
u_{x y}=F\left(u_{x}\right)
$$

and the transformations are given by

$$
\begin{aligned}
& x=a t+b, \\
& y=c z+d, \\
& u=t+S(z)+r w,
\end{aligned}
$$

for some arbitrary constants $a, b, c, d, q, r$, with $a c r \neq 0$, and arbitrary function $S=S(z)$. The corresponding transformed equation has the following expression:

$$
w_{t z}=H\left(w_{t}\right)
$$

where $H=(a c / r) F\left((q / a)+(r / a) w_{t}\right)$.

Case 3. $\pi_{1} \pi_{2} \neq 0$. The equivalence transformations are given by

$$
\begin{aligned}
& x=a t+b, \\
& y=c z+d, \\
& u=\frac{q}{\pi_{1}}+p e^{-\left(a \pi_{1} t\right) / \pi_{2}}+r w,
\end{aligned}
$$

for some arbitrary constants $a, b, c, d, q, p, r$, with acr $\neq 0$. The corresponding transformed equation has the following expression:

$$
w_{t z}=H\left(w_{t}\right)
$$

where $H=(a c / r) F\left(q+\pi_{1} r w+\left(\pi_{2} r / a\right) w_{t}\right)$

Proof. The three cases outlined in the statement of the theorem clearly exhaust all possibilities for the nonconstant function $F$. To establish the stated result in Case 1 , we subject (2a) to the most general invertible point transformation:

$$
\begin{aligned}
& x=\sigma(t, z, w), \\
& y=\tau(t, z, w), \\
& u=\rho(t, z, w),
\end{aligned}
$$

and request that the resulting transformed equation be of the form (2d) for some arbitrary function $H$.

In an expression that depends polynomially on a dependent variable and its derivatives, let us assign to each monomial a weight equal to the total order of derivatives in the 
monomial. In this way, $w_{t z}$ and $w_{t} w_{z z}$ for instance have a weight of two and three, respectively. It turns out that the transformed version of (2a) under (5) will conserve its maximal weight as it should if and only if $\sigma_{w}=\tau_{w}=0$. Therefore, by requesting that the transformed version of (2a) be of the same form, that is an equation of the form (2d), the resulting constraints on the functions $\sigma, \tau$, and $\rho$ reduce the admissible transformations of $x, y$, and $u$ precisely to (2b) or (2c), and the transformed equation to $(2 \mathrm{~d})$ with the specified expression for $H$. The proof is similar for Case 2 and Case 3. This completes the proof.

Due to the properties of invertible point transformations, it is clear that in Theorem 1, the functions $F$ in the original equation and $H$ in its transformed version are related by an equivalence relation. Such an equivalence relation will be denoted $F \sim H$. In particular, in Case 3 , where $\pi_{1} \pi_{2} \neq 0$, we may assume that $F=F\left(u+u_{x}\right)$ in (1).

\section{Lie Group Classification}

The group classification of (1) will be performed under point transformations. We denote by $\mathbf{v}=\xi \partial_{x}+\eta \partial_{y}+\phi \partial_{u}$, a generic symmetry vector of (1), where $\xi, \eta$, and $\phi$ are functions of $x, y$, and $u$. By generic symmetry vector of (1), we mean a linear combination of all symmetry generators of (1).

The determining equations for the symmetries of (1) whose expression is omitted here show that the conditions

$$
\xi_{u}=\xi_{y}=\eta_{u}=\eta_{x}=\phi_{u u}=\phi_{x u}=0
$$

should hold. Consequently,

$$
\begin{aligned}
& \xi=\xi(x), \\
& \eta=\eta(y), \\
& \phi=g u+h,
\end{aligned}
$$

for some functions $g=g(y)$ and $h=h(x, y)$. The remaining part of the determining equations reduces to the classifying equation:

$$
\begin{aligned}
0= & -g \pi_{1} u F^{\prime}-F^{\prime}\left(h \pi_{1}+\pi_{2} h_{x}\right)+F\left(g-\eta_{y}-\xi_{x}\right) \\
& +u_{x}\left[g_{y}+\pi_{2} F^{\prime}\left(-g+\xi_{x}\right)\right]+h_{x y}
\end{aligned}
$$

where here and in the sequel, the notation $f^{\prime}$ denotes the derivative of the function $f$ of a single variable with respect to its argument.

Our general procedure for the group classification problem goes as follows. In each term of (7), we consider as indeterminate in a polynomial expression those factors involving only functions of $u$ and its derivatives. In this way the vanishing coefficients of the polynomial expression can be unambiguously determined if we know exactly which of the indeterminates are nontrivially linearly dependent and which ones are not. Here, a nontrivial linear dependence is one for which all coefficients in the corresponding vanishing linear combination are nonzero.

When the expression of the determining equations can take the form of a polynomial equation in which the indeterminate involves the labelling parameters (of the family of equations) having as arguments variables which are by themselves indeterminate for the same determining equations, extensions of the principal algebra will occur if and only if one or more indeterminates are linearly dependent. The linear dependence of a single indeterminate will indeed be reduced simply to its vanishing.

Therefore, once the principal algebra has been determined in the usual way by treating all labelling parameters as arbitrary, to find any of its extension, we look for all possible nontrivially linearly dependent indeterminates. Each linear dependence of an indeterminate will generally reduce the degree of freedom of the labelling parameter, and its new value thus obtained will be inserted in the determining equations and either yield a complete determination of the corresponding symmetry algebra, or a recursive application of the process which will always end up giving a completely determined value of the parameters. In this way the complete list of nonequivalent symmetry classes of the equation is found.

To determine all possible sets of linearly dependent indeterminates, we consider all possible subsets of $m$ indeterminates, where $2 \leq m \leq n$, assuming that there is a total of $n$ indeterminates in the (single scalar) classifying equations. For each such subset of indeterminates, the linear dependence will be determined either by the direct vanishing of the linear combination of the $m$ indeterminate or equivalently by the vanishing of their Wronskian, as the labelling parameter functions are assumed to be smooth. In other words, for each value of $m$, there will be at most $\left(\begin{array}{l}n \\ m\end{array}\right)=n$ ! $/[(n-m) ! m !]$ valid determining equations for the labelling parameters. As for the possible vanishing of a single indeterminate, this will usually be checked by mere inspection, and corresponds indeed to having $m=1$.

Given the conditions on the function $F$ in (1), we will treat separately three cases.

3.1. Case 1: $F=F(u)$. This case amounts to having $\pi_{2}=0$ in (1). Updating (7) with this expression for $F$ shows that $g_{y}=0$ must hold. Hence, $g=A$ must be a constant. With this value of $g,(7)$ reduces to

$$
-F_{u} h-u F_{u} A+F\left(A-\eta_{y}-\xi_{x}\right)+h_{x y}=0
$$

As a consequence of Theorem 1, in the determination of the group classification of $(2 \mathrm{a})$, one can always replace, in particular, the function $F=F(u)$ by any of its nonzero scalar multiples, although admissible transformations of $F$ are quite more general than this.

For the study of equation (8), we first consider the case where the labelling function $F$ is arbitrary. The vanishing of the coefficient of $F_{u}$ then shows that $h=A=0$, while the vanishing of the coefficient of $F$ yields $\xi_{x}=-\eta_{y}$. 
Consequently, for $F$ arbitrary, the symmetry algebra of (2a) is generated by

$$
\mathbf{v}=\left(k_{1} x+k_{2}\right) \partial_{x}+\left(k 3-k_{1} y\right) \partial_{y}
$$

where here and in the sequel, $k_{j}$ (for $j=1,2, \cdots$ ) represent arbitrary constants.

Assuming now that $F$ is not arbitrary in (8), the set $\mathcal{S}=\left\{F_{u}, u F_{u}, F, 1\right\}$ of the indeterminate consists precisely of $n=4$ elements, none of which may vanish due to the assumption on $F$.

3.1.1. Two Indeterminates Are Linearly Dependent. The constraints on $F$ determined by the linear dependence of all possible subsets of 2 elements from $\mathcal{S}$ are given by

$$
\begin{aligned}
& 0=F_{u}^{2}, \\
& 0=F_{u}^{2}-F F_{u u}, \\
& 0=-F_{u u}, \\
& 0=u F_{u}^{2}-F\left(F_{u}+u F_{u u}\right), \\
& 0=-F_{u}-u F_{u u}, \\
& 0=F_{u},
\end{aligned}
$$

Since by assumption the function $F$ may not assume a constant value, it follows by inspection that (10) yield invalid solutions. Other values of $F$ yielding only the symmetry generator (9) are also to be excluded from the updated list of solutions of (10). The relevant values of $F$ determined by (10) and the corresponding generic symmetry generator $\mathbf{v}$ are therefore given as follows. Here and in the sequel, $a_{j}$ (for $j=1,2, \cdots$ ) are arbitrary constants. Hence, for $F \sim e^{u}$, we have

$$
\mathbf{v}=\xi(x) \partial_{x}+\eta(y) \partial_{y}-\left[\xi_{x}(x)+\eta_{y}(y)\right] \partial_{u},
$$

and for $F \sim u$, we have

$$
\mathbf{v}=\left(k_{1} x+k_{2}\right) \partial_{x}+\left(-k_{1} y+k_{3}\right) \partial_{y}+\left(k_{4} u+h\right) \partial_{u}
$$

where $h$ is a solution of the original equation (2a), while $\xi$ and $\eta$ are arbitrary functions of their arguments.For $F \sim u^{a_{1}}, a_{1}$ $\neq 1$, we have

$$
\mathbf{v}=\left(k_{1} x+k_{2}\right) \partial_{x}+\left(k_{3} y+k_{4}\right) \partial_{y}-\frac{\left(k_{1}+k_{3}\right) u}{a_{1}-1} \partial_{u}
$$

3.1.2. Three Indeterminates Are Linearly Dependent. We now move on to consider the case where three elements in $\mathcal{S}$ are linearly dependent. The conditions on $F$ imposed by all possible such sets of three linearly dependent indeterminates are given as follows:

$$
\begin{aligned}
& 0=-F_{u}^{2} F_{u u}+2 F F_{u u}^{2}-F F_{u} F_{u u u}, \\
& 0=2 F_{u u}^{2}-F_{u} F_{u u u}, \\
& 0=F_{u u}^{2}-F_{u} F_{u u u}, \\
& 0=u F_{u u}^{2}-F_{u}\left(F_{u u}+u F_{u u u}\right) .
\end{aligned}
$$

The change of variables $F=e^{w}$ transforms (12) into

$$
2 w_{u u}^{2}-w_{u} w_{u u u}=0
$$

and the latter has the general solution $w=b_{2} \ln \left(u+b_{1}\right)+b_{3}$, where here and in the sequel, $b_{j}$ (for $j=1,2$ ) denote arbitrary constants. Consequently, the general solution of (12) is given by

$$
F=a_{3}\left(u+a_{1}\right)^{a_{2}} \sim\left(u+a_{1}\right)^{a_{2}} \sim u^{a_{2}},
$$

and thus, this case coincides with the solved case (11c). The other equations in (12) are solved in a way more or less similar to that for equation (12). However, they yield solutions such as

$$
\begin{aligned}
F & =a_{2} \ln \left(u+a_{1}\right)+a_{3}, \\
\text { or } F & =\frac{a_{2} e^{a_{1} u}}{a_{1}}+a_{3}, \\
\text { or } F & =\frac{a_{2} u^{a_{1}}}{a+1}+a_{3},
\end{aligned}
$$

which are often more general solutions than those found for $m=2$, but which in any case do not yield symmetries other than those given by (22).

3.1.3. Four Indeterminates Are Linearly Dependent. We now consider the case where there are four linearly dependent elements in $\mathcal{S}$. There is only one such possibility consisting of the whole set $\mathcal{S}$, and the corresponding constraint on $F$ is given as follows:

$$
F_{u u}^{2} F_{u u u}-2 F_{u} F_{u u u}^{2}+F_{u} F_{u u} F_{u u u u}=0
$$

Setting $F=\int G(u) d u$ and then $G=e^{w(u)}$ reduces (16) to

$$
-2 w_{u u}^{2}+w_{u} w_{u u u}=0
$$

so that $w=c_{3}+c_{2} \ln \left(u+c_{1}\right), G=b_{3}\left(u+b_{1}\right)^{b_{2}}$, for some arbitrary constants $c_{j}, b_{j}$, where $j=1, \cdots, 3$. Consequently, for $b_{2}$ $\neq-1, F \sim\left(u+a_{1}\right)^{a_{2}}+a_{3}$. We note that $F$ is linear in this case if and only if $a_{2}=1$, in which case $F \sim u$ corresponds to the already solved case (11b). On the other hand, for $a_{2} \neq 1$ and $a_{3}=0, F$ corresponds to the solved case (14), while for $a_{2} \neq$ 1 , and $a_{3} \neq 0, F$ yields the symmetry (9) corresponding to arbitrary functions.

Similarly, for $b_{2}=-1$ in the expression of $G=b_{3}$ $\left(u+b_{1}\right)^{b_{2}}$, one gets $F \sim \ln \left(u+a_{1}\right)+a_{2}$, which as already seen, also yields a symmetry generator given by (9). 
Theorem 2. Denote by $L$ the symmetry algebra of the hyperbolic equation $u_{x y}=F(u)$ and by $\mathbf{v}$ the generic symmetry vector in $L$.

(a) For $F(u) \sim u, L$ is infinite dimensional and

$$
\mathbf{v}=\left(k_{1} x+k_{2}\right) \partial_{x}+\left(-k_{1} y+k_{3}\right) \partial_{y}+\left(k_{4} u+h\right) \partial_{u},
$$

where $h$ is a solution of the original equation (2a)

(b) For $F(u) \sim e^{u}, L$ is infinite dimensional and

$$
\mathbf{v}=\xi(x) \partial_{x}+\eta(y) \partial_{y}-\left[\xi_{x}(x)+\eta_{y}(y)\right] \partial_{u}
$$

(c) For $F(u) \sim u^{a_{1}}$, with $a_{1} \neq 1$, L has dimension 4 and

$$
\mathbf{v}=\left(k_{1} x+k_{2}\right) \partial_{x}+\left(k_{3} y+k_{4}\right) \partial_{y}-\frac{\left(k_{1}+k_{3}\right) u}{a_{1}-1} \partial_{u}
$$

(d) For any other function $F(u)$, L has dimension 3 and

$$
\mathbf{v}=\left(k_{1} x+k_{2}\right) \partial_{x}+\left(k 3-k_{1} y\right) \partial_{y}
$$

The four symmetry classes thus obtained are pairwise nonequivalent and make up all possible symmetry classes of (2a).

Proof. Statement (a), statement (b), statement (c), and statement (d) as well as the fact that the listed symmetry classes exhaust all possible symmetry classes of (2a) are just a summary of the results established immediately before the theorem, and it only remains to show that the stated symmetry classes are nonequivalent. In view of the different dimensions of the symmetry classes found, the only problem is to prove the nonequivalence in the two infinite dimensional cases. However, this follows from the fact that the corresponding functions, namely, $F=e^{u}$ and $F=u$, are clearly nonequivalent under (2b).

3.2. Case 2: $F=F\left(u_{x}\right)$. This case amounts to having $\pi_{1}=0$ in (1), and the resulting expression of the determining equations (7) is reduced to the single equation:

$$
u_{x} g_{y}+F\left(g-\eta_{y}-\xi_{x}\right)+u_{x} F_{u_{x}}\left(\xi_{x}-g\right)-F_{u_{x}} h_{x}+h_{x y}=0,
$$

which will be used to classify (6a) and (6b).

When the function $F$ in (22) is arbitrary and correspondingly $m=0$, an analysis similar to the one done for the preceding case $F=F(u)$ shows that the generic symmetry is given by $\mathbf{v}=V_{0,1}$, where

$$
V_{0,1}=\left(k_{1} x+k_{2}\right) \partial_{x}+k_{3} \partial_{y}+\left(k_{1} u+P\right) \partial_{u}
$$

and $P=P(y)$ is an arbitrary function. It is a remarkable fact that the class (3a) of equations has an infinite dimensional principal symmetry algebra. It might therefore be possible that this class of equations which by the way are generally easily integrable, is linearizable by certain types of transformations, although it clearly follows from Theorem 1 that (3a) is not linearizable by point transformations.

For the rest of the classification of (3a), we continue with the application of our method based on the determination of all possible cases of linearly dependent subsets of $m$ indeterminates in (22), with $2 \leq m \leq n$, where in this case $n=5$ is the cardinality of the set $\mathcal{S}=\left\{u_{x}, F, u_{x} F_{u_{x}}, F_{u_{x}}, 1\right\}$ of indeterminates. In the set of equations representing the constraints on $F$ for a particular value of $m$, only those equations corresponding to new and nonredundant solutions will usually be represented.

3.2.1. Two Indeterminates Are Linearly Dependent. For $m=2$, the constraints on $F$ are given by

$$
\begin{aligned}
20 & =-F+u_{x} F_{u_{x}}, \\
0 & =-F_{u_{x}}+u_{x} F_{u_{x} u_{x}}, \\
0 & =u_{x}^{2} F_{u_{x} u_{x}}, \\
0 & =-F_{u_{x}}^{2}+F F_{u_{x} u_{x}}, \\
0 & =-u_{x} F_{u_{x}}^{2}+F\left(F_{u_{x}}+u_{x} F_{u_{x} u_{x}}\right), \\
0 & =-F_{u_{x}}-u_{x} F_{u_{x} u_{x}} .
\end{aligned}
$$

To simplify notation, we will make use of the following vector fields, where here and in the sequel, unless otherwise stated, $\xi$ and $H$ are arbitrary functions of $x$, while $g$ and $P$ are arbitrary functions of $y$. Also, $S=S(x, y)$ is a particular solution of the linear equation $u_{x, y}-u_{x}=0$, and $\alpha$ is an arbitrary constant.

More precisely, for each value of $m$, we denote by $V_{m, j}$ the $j$ th generic generator, as they consecutively occur, of the symmetry class associated with a solution of an ode representing the condition of linear dependence of $m$ indeterminates. Therefore, let us set

$$
V_{2,1}=\xi \partial_{x}+\left(k_{1}+g\right) \partial_{y}+[g u+S(x, y)] \partial_{u}
$$

$$
\begin{aligned}
V_{2,2}= & \left(k_{5}+k_{6} x\right) \partial_{x}+\left(k_{2}+y\left(k_{3}+k_{4} y\right)\right) \partial_{y} \\
& +\left[k_{1}+P-k_{4} x+u\left(-k_{3}+k_{6}-2 k_{4} y\right)\right] \partial_{u},
\end{aligned}
$$

$$
\begin{aligned}
V_{2,3}= & \left(k_{1}+k_{2} x\right) \partial_{x}+\frac{2 k_{5}-k_{4} \cos (2 y)+k_{3} \sin (2 y)}{2} \partial_{y} \\
& +\left[P+k_{2} u-\left(k_{3} u+k_{4} x\right) \cos (2 y)+\left(-k_{4} u+k_{3} x\right) \sin (2 y)\right] \partial_{u}
\end{aligned}
$$

$V_{2,4}=\left(k_{3}+k_{4} x\right) \partial_{x}+\left(k_{1}+k_{2} y\right) \partial_{y}+\left[P+u\left(k_{4}+\frac{k_{2}}{1-\alpha}\right)\right] \partial_{u}$, 


$$
V_{2,5}=\left(k_{2}+k_{3} x\right) \partial_{x}+\left(k_{1}-k_{5} y\right) \partial_{y}+\left(k_{4}+P+k_{3} u+k_{5} x\right) \partial_{u} .
$$

The solutions $F$ of (24) represented by their canonical forms under (3b) and the corresponding generic symmetry vector $\mathbf{v}$ are given as follows:

$$
\begin{gathered}
2 F \sim u_{x}, \\
\mathbf{v}=V_{2,1}, \\
F \sim u_{x}{ }^{2}, \\
\mathbf{v}=V_{2,2}, \\
F \sim u_{x}{ }^{2}+\alpha \sim u_{x}{ }^{2}+1, \quad(\alpha \neq 0), \\
\mathbf{v}=V_{2,3}, \\
(\alpha \notin\{1,2\}), \\
\mathbf{v}=V_{2,4}, \\
F \sim e^{u_{x}}, \\
\mathbf{v}=V_{2,5} .
\end{gathered}
$$

3.2.2. Three Indeterminates Are Linearly Dependent. In order to write down more concisely the set of conditions on $F$ corresponding to $m=3$, we set $u_{x}=v$. The equations are then given by

$$
\begin{aligned}
& 0=F_{v v v}, \\
& 0=-F_{v v}^{2}+F_{v} F_{v v v}, \\
& 0=-F_{v}^{2} F_{v v}+2 F F_{v v}^{2}-F F_{v} F_{v v v} \\
& 0=-v F_{v v}^{2}-F F_{v v v}+F_{v}\left(F_{v v}+v F_{v v v}\right), \\
& 0=-F\left(2 F_{v v}+v F_{v v v}\right)+v\left(-v F_{v v}^{2}+F_{v}\left(2 F_{v v}+v F_{v v v}\right)\right) .
\end{aligned}
$$

In view of the equivalence transformations (2b) and the linear case already treated, one may consider that the solution $F$ of (27) satisfies $F \sim u_{x}^{2}+\beta u_{x}+\alpha$. It turns out that $F$ $\sim u_{x}^{2}$ when $\beta^{2}-4 \alpha=0$, and $F \sim u_{x}^{2}+1$ if otherwise. Since these two cases already appear in (26), it then follows that (27) does not yield any new result.

The general solution $F$ of (27) satisfies $F \sim e^{u_{x}}+\beta$, and for $\beta=0$, the corresponding symmetry is given by (26), while for $\beta \neq 0, F \sim e^{u_{x}}+1$ and the corresponding symmetry is given by $\mathbf{v}=V_{3,1}$, where

$$
V_{3,1}=\left(k_{1}+k_{2} x\right) \partial_{x}+\left(k_{4}-k_{3} e^{-y}\right) \partial_{y}+\left(P(y)+k_{2} u-k_{3} x e^{-y}\right) \partial_{u} .
$$

The next equation to consider from (27) is (27) which is up to a renaming of the unknown function's argument the same as (12) already solved and whose solution satisfies $F \sim\left(\alpha+u_{x}\right)^{\beta}$. The symmetries corresponding to the solution $F$ are given as follows for $\alpha \neq 0$, the case $\alpha=0$ being already solved in (26).

$$
\begin{aligned}
2 F & \sim u_{x}^{2}, \\
\mathbf{v} & =V_{2,2}, \\
F & \sim u_{x}^{\beta}, \quad(\beta \notin\{1,2\}), \\
\mathbf{v} & =V_{2,4},
\end{aligned}
$$

where $V_{2,2}$ and $V_{2,4}$ are the symmetries already found in (26). Hence (27) does not yield any new result either.

Up to this point, the constraints on $F$ resulting from the condition of linear dependence of indeterminates have been expressed typically as $m$ th order odes, and we have luckily been able to solve all such odes. However, some of them, such as (27), are hard to solve and we have to resort to the more direct method of expressing the condition of linear dependence as the vanishing of a linear combination of the indeterminate with some arbitrary constant coefficient. In that way all such constraints turn out in this instance to be mere linear first order odes. The drawback with this method lies in the rigidity of arbitrary constants in the resulting general solutions which albeit straightforward to find, are somewhat much harder to reduce to a simpler and suitable form. This is due to the fact that the coefficients in the linear combination are in fact also arbitrary parameters of the required solution.

For equation (27), the corresponding vanishing of the linear combination of indeterminate takes the form

$$
0=\alpha u_{x}+\beta F+\sigma F_{u_{x}}
$$

with solution

$$
F=\delta e^{-\left(\beta u_{x} / \sigma\right)}-\alpha \frac{\left(-\sigma+\beta u_{x}\right)}{\beta^{2}},
$$

where the constant of integration $\delta$ together with the coefficient $\alpha, \beta$, and $\tau$ are to be considered as arbitrary parameters in the solution (30b). With a bit of manipulation, the latter solution can be put into the form

$$
F=\lambda\left(1+\beta u_{x}\right)+\sigma e^{\beta u_{x}}
$$

where the parameters $\beta, \sigma$, and $\lambda$ are different from those in (30b). In fact, the renaming of parameters in a transformed expression to those appearing in the original expression will often be assumed in the sequel. Applying now the equivalence relation $(3 c)$ to the latter expression for $F$ reduces it to the form $F \sim \beta e^{u}+u$ for some new arbitrary constant $\beta$. Finally inserting the latter expression for $F$ into the determining equations (22) and solving shows that the only existing symmetries for equation (27) are those for $F$ arbitrary and given by (23).

The symmetries corresponding to the third order ODE (27) are found with the same procedure as above for (27). The linear dependence constraints on $F$ takes the form

$$
0=\alpha u_{x}+\beta F+\sigma u_{x} F_{u_{x}}
$$


with the solution

$$
F=\delta u_{x}^{-(\beta / \sigma)}-\frac{\alpha u_{x}}{\beta+\sigma},
$$

Thanks to the equivalence relation (3c), one has $F \sim u_{x}$ $+u_{x}^{\beta}$, and substituting the latter expression into (22) gives rise to two relevant cases to consider, namely, the case $\beta=2$ for which $F \sim u_{x}^{2}+1$ correspond to a case already solved, and the more general case given as follows together with its corresponding generic symmetry.

$$
\begin{aligned}
2 F & \sim u_{x}^{\beta}+u_{x}, \quad(\beta \notin\{1,2\}), \\
\mathbf{v} & =V_{3,2},
\end{aligned}
$$

where

$V_{3,2}=\left(k_{1}+k_{2} x\right) \partial_{x}+\left[k_{4}+\frac{k_{3} e^{y(1-\beta)}}{1-\beta}\right] \partial_{y}+\left[k_{2}+\frac{k_{3} e^{y(1-\beta)}}{1-\beta} u\right] \partial_{u}$.

3.2.3. Four Indeterminates Are Linearly Dependent. Assuming now that $m=4$ of the indeterminate in (22) are linearly dependent gives rise to a maximum of four possible conditions on the function $F$, only two of which yield meaningful solutions and are given as follows:

$$
\begin{aligned}
0= & -F_{v}^{2} F_{v v}+2 F F_{v v}^{2}-F F_{v} F_{v v v}, \\
0= & -v F_{v v}^{2} F_{v v v}-3 F F_{v v v}^{2}+2 F F_{v v} F_{v v v v}+F_{v} \\
& \cdot\left(2 v F_{v v v}^{2}+F_{v v}\left(F_{v v v}-v F_{v v v v}\right)\right) .
\end{aligned}
$$

Equation (35) is just equation (16) whose general solution in terms of $u_{x}$ satisfies $F \sim u_{x}^{\alpha}+\beta$. In view of the preceding cases, we have to assume $\beta \neq 0$, and hence, the resulting relevant cases are given by $F \sim u_{x}^{\alpha}+\beta$ (arbitrary), and $F \sim$ $u_{x}^{3}+\beta$ which all yield no new symmetries apart from the one given by (23). The other relevant case is $F \sim u_{x}^{2}+\beta \sim u_{x}^{2}$ +1 which is also an already solved case. In other words, equation (35) yields no new symmetries.

On the other hand, Equation (35) has general solution:

$$
F=\lambda\left(\tau+u_{x}\right)^{\beta}+\frac{\alpha\left(\tau+\beta u_{x}\right)}{\beta-1},
$$

and by $(3 \mathrm{c})$, one has

$$
F \sim \delta u_{x}^{\beta}+u_{x}+\alpha(\alpha, \beta, \delta \neq 0) .
$$

In view of the preceding results, the latter value for $F$, and hence Equation (35), yields no new symmetries.

3.2.4. Five Indeterminates Are Linearly Dependent. For the last case in (22) where there are five linearly dependent indeterminates in $u_{x}$, the corresponding single equation is given by

$$
0=-F_{v v v}^{2} F_{v v v v}+2 F_{v v} F_{v v v v}^{2}-F_{v v} F_{v v v} F_{v v v v v} .
$$

Setting $F=\iint G(v) d v d v$, and then $G=e^{w(v)}$ reduces (37) to the simpler equation $2 w_{v v}^{2}-w_{v} w_{v v v}=0$, whose solution is $w=\beta \ln (\alpha+v)+\delta$. However, none of the corresponding solutions of the original equation (37) yields a new symmetry.

Theorem 3. For the hyperbolic equation $u_{x y}=F\left(u_{x}\right)$, the list of all possible nonequivalent values of $F=F\left(u_{x}\right)$ yielding, under point transformations, nonequivalent symmetry algebras with generic vectors $\mathbf{v}$ are given by Tables 1 and 2, and consists of a total of eight nonequivalent symmetry classes.

Proof. The fact that Tables 1 and 2 list all possible nonequivalent or distinct values of $F$ and the corresponding symmetry algebras follows from the discussion carried out in Section 3.2. The simplification of the canonical form of each function $F$ listed in the table naturally follows from (3c). However, it remains to be ascertained if all functions in these lists are pairwise nonequivalent. This also follows by a straightforward application (3c). This completes the proof.

It should be noted that by using a simple symmetry argument based on the structure of the resulting equation $u_{x y}=$ $F\left(u_{x}\right)$ with respect to the variables $x$ and $y$, the results from this section implicitly include those corresponding to the case $F=F\left(u_{y}\right)$.

3.3. Case 3: $F=F\left(u+u_{x}\right)$. As already noted, in view of (1), this case corresponds to having $\pi_{1} \pi_{2} \neq 0$ in (1). The corresponding determining equation (7) then reduces to

$$
\begin{aligned}
0= & -g u F^{\prime}-F^{\prime}\left(h+h_{x}\right)+F\left(g-\eta_{y}-\xi_{x}\right) \\
& +u_{x}\left[g_{y}+F^{\prime}\left(-g+\xi_{x}\right)\right]+h_{x y} .
\end{aligned}
$$

When $F$ is arbitrary in (38), it readily follows that the functions $\xi, \eta$, and $\phi$ from (6a) and (6b) are given by $\xi=k_{1}$, $\eta=k_{2}$, and $\phi=e^{-x} k_{3}$, for some arbitrary constants $k_{1}, k_{2}$, and $k_{3}$. In other words, the principal algebra has a generic vector:

$$
\mathbf{v}=k_{1} \partial_{x}+k_{2} \partial_{y}+k_{3} e^{-x} \partial_{u}
$$

Given that in (38) $F$ is a function of $\vartheta=u+u_{x}$ while (38) itself depends explicitly on $u$ and $u_{x}$, in order to apply our classification procedure for the extension of the principal algebra to (38), it is more appropriate to first transform the whole of (1) under the new dependent variable:

$$
\vartheta=u+u_{x}
$$

which turns out to be invertible, within verse

$$
u=\vartheta-\int F(\vartheta) d y,
$$


TABLE 1: Nonequivalent symmetry classes for $u_{x y}=F\left(u_{x}\right)$ : first four classes. The $k_{j}$ are the free parameters of the symmetry group. The functions $\xi=\xi(x), H=H(x), P=P(y), g=g(y)$ are arbitrary while $S=S(x, y)$ is a particular solution of the linear equation $u_{x y}=u_{x}$ and $\alpha$, $\beta, \sigma$, and $\delta$ are free parameters defining the function $F$.

\begin{tabular}{lc}
\hline Numbering & Canonical function $F_{j}$ and generic symmetry vector $\mathbf{v}_{j}$, for $j=1, \cdots, 4$ \\
\hline$F_{1}$ & $u_{x}$ \\
$\mathbf{v}_{1}$ & $\xi \partial_{x}+\left(k_{1}+g\right) \partial_{y}+[g u+S(x, y)] \partial_{u}$ \\
$F_{2}$ & $u_{x}^{2}$ \\
$\mathbf{v}_{2}$ & $\left(k_{5}+k_{6} x\right) \partial_{x}+\left(k_{2}+y\left(k_{3}+k_{4} y\right)\right) \partial_{y}+\left[k_{1}+P-k_{4} x+u\left(-k_{3}+k_{6}-2 k_{4} y\right)\right] \partial_{u}$ \\
$F_{3}$ & $\left(k_{1}+k_{2} x\right) \partial_{x}+\frac{2 k_{5}-k_{4} \cos (2 y)+k_{3} \sin (2 y)}{2} \partial_{y}+\left[P+k_{2} u-\left(k_{3} u+k_{4} x\right) \cos (2 y)+\left(-k_{4} u+k_{3} x\right) \sin (2 y)\right] \partial_{u}$ \\
$\mathbf{v}_{3}$ & $u_{x}^{\alpha}, \alpha \notin\{1,2\}$ \\
$F_{4}$ & $\left(k_{3}+k_{4} x\right) \partial_{x}+\left(k_{1}+k_{2} y\right) \partial_{y}+\left[P+u\left(k_{4}+\frac{k_{2}}{1-\alpha}\right)\right] \partial_{u}$ \\
$\mathbf{v}_{4}$ &
\end{tabular}

TABLE 2: Nonequivalent symmetry classes for $u_{x y}=F\left(u_{x}\right)$ : last four classes. The $k_{j}$ are the free parameters of the symmetry group. The functions $\xi=\xi(x), H=H(x), P=P(y), g=g(y)$ are arbitrary, while $S=S(x, y)$ is a particular solution of the linear equation $u_{x y}=u_{x}$ and $\alpha$ $, \beta, \sigma$, and $\delta$ are free parameters defining the function $F$.

\begin{tabular}{lc}
\hline Numbering & Canonical function $\left(F_{j}\right)$ and generic symmetry vector $\left(\mathbf{v}_{j}\right)$ \\
\hline$F_{5}$ & $e^{u_{x}}$ \\
$\mathbf{v}_{5}$ & $\left(k_{2}+k_{3} x\right) \partial_{x}+\left(k_{1}-k_{5} y\right) \partial_{y}+\left(k_{4}+P+k_{3} u+k_{5} x\right) \partial_{u}$ \\
$F_{6}$ & $e^{u_{x}}+1$ \\
$\mathbf{v}_{6}$ & $\left(k_{1}+k_{2} x\right) \partial_{x}+\left(k_{4}-k_{3} e^{-y}\right) \partial_{y}+\left(P(y)+k_{2} u-k_{3} x e^{-y}\right) \partial_{u}$ \\
$F_{7}$ & $u_{x}^{\beta}+u_{x},(\beta \notin\{1,2\})$ \\
$\mathbf{v}_{7}$ & $\left(k_{1}+k_{2} x\right) \partial_{x}+\left[k_{4}+\left(k_{3} / 1-\beta\right) e^{y(1-\beta)}\right] \partial_{y}+\left[P+k_{2}+\left(k_{3} / 1-\beta\right) e^{y(1-\beta)} u\right] \partial_{u}$ \\
$F_{8}$ & $F$ is arbitrary \\
$\mathbf{v}_{8}$ & $\left(k_{1} x+k_{2}\right) \partial_{x}+k_{3} \partial_{y}+\left(k_{1} u+P\right) \partial_{u}$ \\
\hline
\end{tabular}

and rather apply the classification procedure for the determination of all admissible functions $F$ to the resulting transformed equation. Indeed, since (40a) and (40b) is invertible, there is a one-to-one correspondence between any function $F$ associated with (1) and a function $F$ associated with the transformed version of (1) under (40a) and (40b) whose explicit expression takes the form

$$
\vartheta_{x y}=F(\vartheta)+\vartheta_{x} F^{\prime}(\vartheta)
$$

Denoting by $\mathbf{w}=\mu \partial_{x}+\nu \partial_{y}+\psi \partial_{\vartheta}$ the generic symmetry generator in the symmetry algebra of (41), the associated determining equations show that

$$
\begin{aligned}
& \mu=\mu(x), \\
& \nu=v(y), \\
& \zeta=\zeta(y) \vartheta+\omega(x, y),
\end{aligned}
$$

for some arbitrary functions $\zeta$ and $\omega$. Moreover, the remaining determining equations take the form

$$
\begin{aligned}
& 0=\zeta_{y}-F^{\prime} v_{y}-\omega F^{\prime \prime}-\zeta \vartheta F^{\prime \prime}, \\
& 0=\zeta_{y}-F^{\prime}\left(\omega+\omega_{x}+v_{y}\right)+F\left(\zeta-v_{y} \xi_{x}\right)-\omega F^{\prime \prime}-\zeta \vartheta\left(F^{\prime}+F^{\prime \prime}\right)+\omega_{x, y} .
\end{aligned}
$$

As far as the determination of an indeterminate in (43) is concerned, only equation (43) may be considered, as it contains all the indeterminate in (43). Hence, the list of indeterminates for (41) is given by

$$
\ell=\left\{1, F, F^{\prime}, F^{\prime \prime}, \vartheta\left(F^{\prime}+F^{\prime \prime}\right)\right\}
$$

The admissible functions corresponding to this list and extending the principal algebra of (41) are by construction the same as those extending the principal algebra of the original equation 


$$
u_{x y}=F\left(u+u_{x}\right)
$$

Finding these admissible functions the usual way as in Subsections 3.1 and 3.2 yield the following complete classification of (45).

Theorem 4. Denote by $L$ the symmetry algebra of the hyperbolic equation (45), which is equivalent under (4a) to $u_{x y}=$ $F\left(\pi_{1} u+\pi_{2} u_{x}\right)$ with $\pi_{1} \pi_{2} \neq 0$, and denote by $v$ the generic symmetry vector in $L$.

(a) For $F\left(u+u_{x}\right) \sim u+u_{x}, L$ is infinite dimensional and

$$
\mathbf{v}=\left(k_{2}-k_{4} x\right) \partial_{x}+\left(k_{1}+k_{4} y\right) \partial_{y}+\left[S(x, y)+\left(k_{3}+k_{4} y\right) u\right] \partial_{u},
$$

where $S(x, y)$ is any solution of the linear equation $u_{x y}=u+$ $u_{x}$

(b) For $F\left(u+u_{x}\right) \sim e^{u+u_{x}}, L$ is infinite dimensional and

$$
\mathbf{v}=k_{1} \partial_{x}+\eta(y) \partial_{y}+\left[k_{2} e^{-x}-\eta_{y}(y)\right] \partial_{u}
$$

(c) For $F\left(u+u_{x}\right) \sim\left(u+u_{x}\right)^{\alpha}$, with $\alpha \neq 1$, L has dimension 4 and

$$
\mathbf{v}=k_{1} \partial_{x}+\left(k_{2}+k_{3} y\right) \partial_{y}+\left(k_{4} e^{-x}+\frac{k_{3} u}{1-\alpha}\right) \partial_{u}
$$

(d) For any other function $F\left(u+u_{x}\right)$, L has dimension 3 and

$$
\mathbf{v}=k_{1} \partial_{x}+k_{2} \partial_{y}+k_{3} e^{-x} \partial_{u}
$$

The four symmetry classes thus obtained are pairwise nonequivalent and make up all possible symmetry classes of (45).

Proof. The proof is similar to that given for Theorem 2 and Theorem 3, and the details are omitted.

\section{Conservation Laws}

As discussed in Section 1, the many physical applications associated with several equations of the form (1) motivates the study in this section of conservation laws of this class of equations. Let us recall that a conservation law of (1) is a divergence expression:
$\operatorname{Div} \Theta \equiv D_{x} \Phi+D_{y} \Psi=0, \quad \Theta=\Theta[u]=(\Phi, \Psi)=(\Phi[u], \Psi[u])$,

that vanishes on the solution space of (1), where $D_{x}$ and $D_{y}$ are the usual total differential operators acting on the jet space of the underlying space $X \times U \mathbb{R}^{3}$ of independent variables $(x, y) \in X$ and dependent variable $u \in U$. Moreover, the notation $f=f[u]$ denotes a differential function of $u$, that is, a function depending on $x, y$, and $u$ and the derivatives of $u$ up to an arbitrary but fixed order. The vector $\Theta=(\Phi, \Psi)$ is then called a conserved current, and may also be called a flux vector, as we shall do.

Conservation laws are also determined up to an equivalence class, whereby two conservation laws are termed equivalent if they differ by a trivial conservation law, that is, by one which vanishes for all smooth functions $u=u(x$ ,$y$ ) and not only on the solution space of (1). Such trivial conservation laws are said to be of the second kind as opposed to trivial conservation laws of the first kind in which the function $\Theta$ itself, in (50), vanishes on all solutions of the equation [21]. Trivial conservation laws of the first kind will be eliminated by the choice of derivatives appearing as arguments in $\Theta$.

To each conservation law (50), there corresponds a multiplier $Q=Q[u]$ given by

$$
Q \cdot \Delta=D_{x} \Phi[u]+D_{y} \Psi[u]=0, \quad \Delta \equiv u_{x y}-F\left(u u_{x}\right),
$$

and two multipliers are equivalent if they differ by a trivial multiplier, that is, by one which vanishes itself on the solution space of the equation. For normal and totally nondegenerate equations of the form (1), there is a one-to-one correspondence between equivalent classes of multipliers and equivalent classes of conservation laws.

For Lagrangian equations, every characteristic of a variational symmetry is a multiplier and can be uniquely identified with a conservation law. However, Lagrangian equations constitute a very restricted type of equation and for more general equations for which the concept of variational symmetry in particular does not apply, multipliers are found as generalized integrating factors. That is, as factors $Q=Q[u]$ for which the product $Q \cdot \Delta$ is a total divergence expression, and hence, a null Lagrangian.

Our search for conservation laws will be focussed on those of low order, as physically relevant properties such as energy, mass-energy, and momentum conservation are always associated with these low-order conservation laws. In mathematical terms, these are conservation laws for which every derivative of a dependent variable in the equation can be obtained by differentiating with respect to some independent variable a similar derivative in the expression of the multiplier.

Conservation laws of (1) with $F=F(u)$ are given for arbitrary values of $F$ in [30], although [30] does not make any reference to the corresponding multiplier. It is indeed possible to find a specific flux vector $\Theta$ directly, albeit generally more intricate, by solving (50) on the solution surface of the equation. We complement the result of [30] by finding 
the multiplier $Q$ which is to be sought in the form $Q=Q(x$, $\left.y, u_{x}\right)$ or $Q\left(x, y, u_{y}\right)$. In view of obvious symmetry considerations associated with the structure of (1), it is enough to search for $Q=Q\left(x, y, u_{x}\right)$. The determining equations for the function $Q$ are as follows:

$$
\begin{gathered}
Q_{u}=0, \\
Q_{y u_{x}}+F Q_{u_{x} u_{x}}=0, \\
F_{u} Q_{u_{x}} u_{x}-Q F_{u}-F Q_{u}+F u_{x} Q_{u u_{x}}+Q_{x y}+F Q_{x u_{x}}+u_{x} Q_{y u}=0,
\end{gathered}
$$

with solution $Q=\alpha u_{x}$, for a certain constant parameter $\alpha$, which we may assume to be equal to 1 thanks to the linearity of the Euler Operator. Indeed, $Q$ is determined by the condition $E_{u}(Q \cdot \Delta)=0$, where $E_{u}$ is the Euler Operator with respect to $u$. The corresponding determining equations for the flux vector $\Theta=(\Phi, \Psi)$ takes the form

$$
\begin{aligned}
0 & =\Phi_{u_{x x}} \\
\Phi_{u_{y y}} & =0 \\
\Psi_{u_{x x}} & =0 \\
\Psi_{u_{y y}} & =0 \\
0 & =u_{x}-\Phi_{u_{y}}-\Psi_{u_{x}}, \\
0=u_{x}\left(-F-\Phi_{u}\right)- & u_{x x} \Phi_{u_{x}}-\Phi_{x}-u_{y} \Psi_{u}-u_{y y} \Psi_{u_{y}}-\Psi_{y} .
\end{aligned}
$$

The latter determining equation has the following solution:

$$
\Theta=\left(-F+\int R_{y} d u+u_{y} R+Z, \frac{u_{x}^{2}}{2}-\int Z_{x} d y-\int R_{x} d u+P-u_{x} R\right),
$$

where $R=R(x, y, u), Z=Z(x, y)$, and $P=P(x)$ are arbitrary functions. It turns out that this flux vector contains as a term a trivial flux, that is, one corresponding to a trivial conservation law, namely, the term

$$
\left(\int R_{y} d u+u_{y} R+Z,-\int Z_{x} d y-\int R_{x} d u+P-u_{x} R\right) .
$$

Using the symmetry argument related to the structure of (1) together with the above results show that the multipliers $Q$ and corresponding conservation laws of (1) are given for $F$ arbitrary by

$$
\begin{aligned}
2 Q & =u_{x}, \\
0 & =D_{x}[-F(u)]+D_{y}\left(\frac{u_{x}^{2}}{2}\right), \\
Q & =u_{y}, \\
0 & =D_{x}\left(\frac{u_{y}^{2}}{2}\right)+D_{y}[-F(u)] .
\end{aligned}
$$

We shall make use of the same procedure used thus far in this section to find the conservation laws of other relevant equations of the form (1). In this way, the next equation from the class (1) with $F=F(u)$ which we consider is the Liouville equation:

$$
u_{x y}=e^{u}
$$

Although the conservation laws of (57), which is a wellstudied equation $[19,20,28]$ are likely to have been computed, we find it interesting to gather all such results in a single short paper. After calculations, the multipliers $Q$ and corresponding conservations laws for (57) are given as follows:

$$
\begin{aligned}
& Q=p_{x}+p u_{x}, \\
& 0=D_{x}\left[-e^{u} p+p_{x} u_{y}\right]+D_{y}\left[-u p_{x x}+\left(\frac{1}{2}\right) p u_{x}^{2}\right], \\
& Q=q_{y}+q u_{y}, \\
& 0=D_{x}\left[-u q_{y y}+\left(\frac{1}{2}\right) q u_{y}^{2}\right]+D_{y}\left[-e^{u} q+q_{y} u_{x}\right],
\end{aligned}
$$

where $p=p(x)$ and $q=q(y)$ are arbitrary functions. This shows in particular that (57) has infinitely many conservation laws of low order. It has in fact been proved, that (58) is symmetry integrable, that is, it has an infinite series of generalized symmetries of arbitrary orders. Moreover [31], all symmetry integrable equations of the form (2a) are given up to scalings and shifts by functions $F=F(u)$ satisfying

$$
\begin{aligned}
F(u) & =e^{u}, \\
F(u) & =e^{u}+e^{-u}, \\
\text { or } F(u) & =e^{u}+e^{-2 u} .
\end{aligned}
$$

Similar integrability properties might also hold for equation (1) with $F=e^{u+u_{x}}$, given that not only such an equation is similar by its type to (2a), but it also satisfies by Theorem 4 , in the same way as (2a) does, the remarkable property of having a symmetry algebra of infinite dimension.

We now let $F(u)=u^{2}+1$ in $(2 \mathrm{a})-(2 \mathrm{~d})$, so that the resulting equation takes the form

$$
u_{x y}=u^{2}+1
$$


One sees that low-order multipliers are linear combinations of $u_{x}$ and $u_{y}$. Letting the multiplier $Q=u_{x}$, the corresponding flux vector has the following expression:

$\Theta=\left(B-u-\frac{u^{3}}{3}+A u_{y}+\int A_{y} d u, C-A u_{x}+\frac{u_{x}^{2}}{2}-\int B_{x} d y-\int A_{x} d u\right)$,

where $A=A(x, y, u), B=B(x, y)$, and $C=C(x)$ are arbitrary functions of their arguments. The pure flux vector associated with the multiplier $Q=u_{x}$, that is, the one deprived of any trivial flux vector is, however, given by

$$
\Theta=\left(-u-\frac{u^{3}}{3}, \frac{u_{x}^{2}}{2}\right)
$$

In other words, the aggregate of arbitrary functions in (61) only yields a trivial flux vector. These results and the symmetrical structure of $(2 \mathrm{a})-(2 \mathrm{~d})$ with respect to the variables $x$ and $y$ show that all multipliers and corresponding conservation laws of (60) are given by

$$
\begin{aligned}
2 Q & =u_{x}, \\
0 & =D_{x}\left(\frac{-u-u^{3}}{3}\right)+D_{y}\left(\frac{u_{x}^{2}}{2}\right), \\
Q & =u_{y}, \\
0 & =D_{x}\left(\frac{u_{y}^{2}}{2}\right)+D_{y}\left(\frac{-u-u^{3}}{3}\right) .
\end{aligned}
$$
order.

In particular, (60) has only two conservation laws of low

For the counterpart (3a)-(3c) of $(2 \mathrm{a})-(2 \mathrm{~d})$ for which $F$ $=F\left(u_{x}\right)$ so that the equation reduces to $u_{x y}=F\left(u_{x}\right)$, no general result of a similar nature concerning the symmetry integrability of this equation seems to be available. To begin with, for arbitrary values of $F$, (3a) has only trivial multipliers and hence trivial conservation laws. Nevertheless, it turns out that for the particular case $F\left(u_{x}\right)=u_{x}^{2}$, the corresponding equation

$$
u_{x y}=u_{x}^{2}
$$

also has an infinity of conservation laws of low order.

Indeed, using our usual procedure described above, the general expression for the multiplier $Q$ of low-order conservation laws of (64) takes the form

$$
Q=T(z, y)+\frac{x}{z^{2}} V\left(x,-\frac{1+z y}{z}\right), \quad z=u_{x}
$$

where $V$ is an arbitrary function of its arguments, and $T=$ $T(z, y)$ is a solution of the second order linear hyperbolic equation

$$
0=2 T+4 z T_{z}+z^{2} T_{z, z}+T_{z y}
$$

Although a general solution of (65b) is not available, we can find its particular solutions, and this will be enough to verify this claim. A practical and relatively simple way to obtain these particular solutions is to use the similarity reduction method yielding group-invariant solutions [21, $32-34]$ (see also $[35,36]$ for more recent applications of this reduction technique). In addition to the solution symmetry $\zeta \partial_{T}$, where $\zeta=\zeta(y, z)$ is any given solution of $(65 \mathrm{~b})$, the other symmetries of this equation are given by

$$
\begin{aligned}
2 \mathbf{v}_{1} & =T \partial_{T}, \\
\mathbf{v}_{2} & =-z \partial_{z}+y \partial_{y}, \\
\mathbf{v}_{3} & =(-2 y z-1) \partial_{z}+y^{2} \partial_{y}+2 T y \partial_{T}, \\
\mathbf{v}_{4} & =\partial_{z} .
\end{aligned}
$$

Group-invariant solutions $T_{1}, T_{2}$, and $T_{3}$ associated with the above symmetries $\mathbf{v}_{1}, \mathbf{v}_{2}$, and $\mathbf{v}_{3}$ of $(65 \mathrm{~b})$ and the corresponding multipliers $Q_{1}, Q_{2}$, and $Q_{3}$ of (64) are given by

$$
\begin{aligned}
2 T_{1} & =\frac{\beta_{1}}{4 u_{x}^{2}}-\frac{\alpha_{1}}{2 u_{x}}, \\
Q_{1} & =T_{1}+\frac{x}{u_{x}^{2}} V, \\
T_{2} & =\frac{\alpha_{2}}{\left(1+y u_{x}\right)^{2}}+\frac{\beta_{2}\left(y u_{x}+\ln \left(y u_{x}\right)\right)}{\left(1+y u_{x}\right)^{2}}, \\
Q_{2} & =T_{2}+\frac{x}{u_{x}^{2}} V, \\
T_{3} & =\frac{\alpha_{3}-y-y^{2} u_{x}}{\beta_{3}\left(1+y u_{x}\right)^{2}} \\
Q_{3} & =T_{3}+\frac{x}{u_{x}^{2}} V,
\end{aligned}
$$

where $u_{x}=z$, the $\alpha_{j}$ and $\beta_{j}$ are arbitrary constants for $j=1$, 2,3 , and the arbitrary function $V$ is given by (65a). Finally, the flux vectors $\Theta_{j}=\left(\Phi_{j}, \Psi_{j}\right)$ corresponding to the multipliers $Q_{j}$ are given by

$$
\begin{aligned}
& \Theta_{1}=\left(\frac{\alpha_{1} u}{2}-\frac{\beta_{1} x}{4},-\frac{1}{4}\left(\frac{\beta_{1}}{u_{x}}+2 \alpha_{1} \ln \left(u_{x}\right)\right)+\int \frac{x}{u_{x}^{2}} V d u_{x}\right), \\
& \Theta_{2}=\left[\frac{-\beta_{2} u}{y}, \frac{\alpha_{2}-2 \beta_{2}}{y}+\int \frac{\alpha_{2}+\beta_{2} u_{x} y+\beta_{2} \ln \left(u_{x} y\right)}{\left(1+u_{x} y\right)^{2}}+\frac{x}{u_{x}^{2}} V d u_{x}\right], \\
& \Theta_{3}=\left[\frac{u}{\beta_{3}}, \frac{\beta_{3}^{-1}}{y}\left(\alpha_{3}-y \ln (y)+y \int\left(\frac{a-y-u_{x} y^{2}}{\left(1+u_{x} y\right)^{2}}+\frac{x}{u_{x}^{2}} V\right) d u_{x}\right)\right] .
\end{aligned}
$$

Clearly, the nontrivial conservation law for each of the pure flux vectors $\Theta_{j}=\left(\Phi_{j}, \Psi_{j}\right)$ above are given by $D_{x} \Phi_{j}+$ $D_{y} \Psi_{j}=0$, and such low-order conservation laws are infinitely 
many as they are dependent on the arbitrary function $V=$ $V(x, p)$, with $p=-\left(1+u_{x} y\right) / u_{x}$.

\section{Concluding Remarks and Future Outlook}

The group classification of differential equations has proved to be a very challenging exercise, even for the simplest types of differential equations that odes represent, and we have made a breakthrough in this paper by finding a simple and systematic way of solving this type of problem, based on the determination of all possible cases of linearly dependent indeterminates in the determining equations. This has allowed us to give a complete classification of the family (1) of equations in a relatively brief manner. Although the method has only been discussed in application to the particular case of Equation (1), it seems, however, quite clear that it can be extended in a straightforward way to at least any scalar differential equation, and in fact to any system of odes or pdes.

It should also be noted that the difficulty with group classification is not really dependent on the dimension of the original equation or of the classifying determining equations, but rather, and with regard in particular to our method, on the number of indeterminate variables which occur themselves as arguments of arbitrary labelling functions. In other words, the classification problem of (1) would be more complicated to solve if one instead had for instance $F=F(u$, $\left.u_{x}, u_{y}\right)$. The full treatment of such cases would give more insight into the method and hopefully point to ways for improvements.

Our method of course caters typically for cases where the arbitrary functions together with their arguments, or the arbitrary parameters viewed as definite functions of given arguments can be treated as indeterminate in the polynomial expression of determining equations. In that case the method is generally self-sufficient and yields the complete classification result. Else it might be used in combination with other methods.

The proposed method could be used to review some of the relevant cases of group classification already appearing in the literature and often cited in this paper, such as those given in papers [13] to [17] for instance. This will entail in particular the consideration of families of equations depending on more than one arbitrary function, each with its own argument, as well as cases where the classifying equations consist of a system of several equations. Moreover, the case of arbitrary labelling functions involving the independent variables should also be considered. Such an undertaking will allow not only to confirm the validity of existing classification results, but also to gain more insight into the method itself.

Calculations done in Section 4 show in particular that Equation (60) has only a finite number of low-order conservation laws, while (57) and (64) have infinitely many of them. However, while (57) is known to be up to two other related cases listed in (59) the only equations of the form (2a) that are symmetry integrable, nothing is known about the symmetry integrability of (64) and in particular about that for the more general case (3a). The symmetry integrability prop- erty of a differential equation remains a very active and challenging domain of research with very limited results, and it would be interesting to find out if (64) is symmetry integrable, and if so, whether such property also relates to the symmetry integrability of the whole family of equations (3a).

\section{Data Availability}

No data were used in this study.

\section{Conflicts of Interest}

The author declares that there is no conflict of interest for this publication.

\section{Acknowledgments}

This work was supported by the NRF Incentive Funding for Rated Researchers grant (Grant Number 97822); and the University of Venda (Grant Number I538).

\section{References}

[1] L. V. Ovsyannikov, "Group Classification of Equations of the Form $y^{\prime \prime}=f(x, y)$," Journal of Applied Mechanics and Technical Physics, vol. 45, no. 2, pp. 153-157, 2004.

[2] F. Güngör, V. I. Lahno, and R. Z. Zhdanov, "Symmetry classification of KdV-type nonlinear evolution equations," Journal of Mathematical Physics, vol. 45, no. 6, pp. 2280-2313, 2004.

[3] S. V. Meleshko and S. Moyo, "On group classification of normal systems of linear second-order ordinary differential equations," Communications in Nonlinear Science and Numerical Simulation, vol. 22, no. 1-3, pp. 1002-1016, 2015.

[4] T. G. Mkhize, S. Moyo, and S. V. Meleshko, "Complete group classification of systems of two linear second-order ordinary differential equations: the algebraic approach," Mathematical Methods in the Applied Sciences, vol. 38, no. 9, pp. 18241837, 2015.

[5] J. C. Ndogmo, "Group classification of a family of secondorder differential equations," Journal of Mathematical Analysis and Applications, vol. 364, no. 1, pp. 242-254, 2010.

[6] Q. Huang, C. Qu, and R. Zhdanov, "Group classification of linear fourth-order evolution equations," Reports on Mathematical Physics, vol. 70, no. 3, pp. 331-343, 2012.

[7] Y. Y. Bagderina, "Symmetries and invariants of the systems of two linear second-order ordinary differential equations," Communications in Nonlinear Science and Numerical Simulation, vol. 19, no. 10, pp. 3513-3522, 2014.

[8] A. Bihlo and R. O. Popovych, "Group classification of linear evolution equations," Journal of Mathematical Analysis and Applications, vol. 448, no. 2, pp. 982-1005, 2017.

[9] P. Basarab-Horwath, V. Lahno, and R. Zhdanov, "The structure of Lie algebras and the classification problem for partial differential equations," Acta Applicandae Mathematica, vol. 69, no. 1, pp. 43-94, 2001.

[10] L. Gagnon and P. Winternitz, "Symmetry classes of variable coefficient nonlinear Schrodinger equations," Journal of Physics A, vol. 26, no. 23, pp. 7061-7076, 1993.

[11] F. Güngör, "Group classification and exact solutions of a radially symmetric porous-medium equation," International Journal of Non-Linear Mechanics, vol. 37, no. 2, pp. 245-255, 2002. 
[12] A. G. Nikitin and R. O. Popovych, "Group classification of nonlinear Schrödinger equations," Ukrainian Mathematical Journal, vol. 53, no. 8, pp. 1255-1265, 2001.

[13] S. Opanasenko, V. Boyko, and R. O. Popovych, "Enhanced group classification of nonlinear diffusion-reaction equations with gradient-dependent diffusivity," Journal of Mathematical Analysis and Applications, vol. 484, no. 1, article 123739, p. 30, 2020.

[14] A. Bihlo, N. Poltavets, and R. O. Popovych, "Lie symmetries of two-dimensional shallow water equations with variable bottom topography," Chaos, vol. 30 , no. 7 , article 073132 , p. 17 , 2020.

[15] O. O. Vaneeva, C. Sophocleous, and P. G. L. Leach, "Lie symmetries of generalized Burgers equations: application to boundary-value problems," Journal of Engineering Mathematics, vol. 91, no. 1, pp. 165-176, 2015.

[16] V. Lahno and R. Zhdanov, "Group classification of nonlinear wave equations," Journal of Mathematical Physics, vol. 46, no. 5, article 053301, 2005.

[17] V. Lahno, R. Zhdanov, and O. Magda, "Group classification and exact solutions of nonlinear wave equations," Acta Applicandae Mathematicae, vol. 91, no. 3, pp. 253-313, 2006.

[18] S. Lie, "Diskussion der Differentialgleichung $d^{2} z / d x d y=F(z)$," Vorlesungen über Minimalflächen, vol. 6, pp. 112-124, 1975, (Reprinted in: S. Lie, Gesammelte Abhandlungen, Vol. 3, B.G. Teubner, Leipzig and H. Aschehoug \& Co, Kristiania, 469478.).

[19] D. Levi, L. Martina, and P. Winternitz, "Structure preserving discretizations of the Liouville equation and their numerical tests," Symmetry, Integrability and Geometry: Methods and Applications SIGMA, vol. 11, p. 80, 2015.

[20] D. Levi, L. Martina, and P. Winternitz, "Lie-point symmetries of the discrete Liouville equation," Journal of Physics A: Mathematical and Theoretical, vol. 48, no. 2, article 025204, 2015.

[21] P. J. Olver, Applications of Lie Groups to Differential Equations, Springer, New York, NY, USA, 1993.

[22] A. R. Adem and B. Muatjetjeja, "Conservation laws and exact solutions for a 2D Zakharov-Kuznetsov equation," Applied Mathematics Letters, vol. 48, pp. 109-117, 2015.

[23] S. C. Anco and A. H. Kara, "Symmetry-invariant conservation laws of partial differential equations," European Journal of Applied Mathematics, vol. 29, no. 1, pp. 78-117, 2018.

[24] S. C. Anco and G. Bluman, "Direct construction method for conservation laws of partial differential equations Part II: General treatment," European Journal of Applied Mathematics, vol. 13, no. 5, pp. 567-585, 2002.

[25] F. Gao and Z. Wang, "Nonlinear self-adjointness and conservation laws for the modified dissipative hyperbolic geometric flow equation," Journal of Geometry and Physics, vol. 2021, article 104304, 2021.

[26] E. Bour, “Théorie de la déformation des surfaces," Journal de l'École Impériale Polytechnique, vol. 19, pp. 1-48, 1862.

[27] J. Frenkel and T. Kontorova, "On the theory of plastic deformation and twinning," Izvestiya Akademii Nauk SSSR, Seriya Fizicheskaya, vol. 1, pp. 137-149, 1939.

[28] B. A. Dubrovin, S. P. Novikov, and A. T. Fomenko, Modern Geometry-Methods and Applications. Part I. The Geometry of Surfaces, Transformation Groups, and Fields, Graduate Studies in Mathematics, 93, Springer Verlag, New York, NY, USA, 2nd edition, 1992.
[29] J. W. Thomas, "Hyperbolic equations," in Numerical Partial Differential Equations: Finite Difference Methods. Texts in Applied Mathematics 22, Springer, 1995.

[30] A. D. Polyanin and V. F. Zaitsev, Handbook of Nonlinear Partial Differential Equations, Second Edition, Chapman and Hall, 2011.

[31] R. Hernández Heredero and V. Sokolov, "The symmetry approach to integrability: recent advances," in Nonlinear Systems and Their Remarkable Mathematical Structures: Vol. 2, N. Euler and M. C. Nucci, Eds., pp. 119-157, CRC Press, 2019.

[32] G. W. Bluman, A. F. Cheviakov, and S. Anco, "Applications of Symmetry Methods to Partial Differential Equations," in Applied Mathematical Sciences, Springer, 2010.

[33] L. V. Ovsyannikov, Group Properties of Differential Equations [in Russian], Novosibirsk, 1962.

[34] H. Stephani, Differential Equations: Their Solution Using Symmetries, Cambridge University Press, 1989.

[35] S. Kumar, A. Kumar, and H. Kharbanda, "Lie symmetry analysis and generalized invariant solutions of $(2+1)$-dimensional dispersive long wave (DLW) equations," Physica Scripta, vol. 95, no. 6, article 065207, 2020.

[36] S. Kumar, W.-X. Ma, and A. Kumar, "Lie symmetries, optimal system and group-invariant solutions of the $(3+1)$-dimensional generalized KP equation," Chinese Journal of Physics, vol. 69, pp. 1-23, 2021. 